\title{
Does health care spending improve health outcomes? Evidence from English programme budgeting data ${ }^{1}$
}

\author{
Stephen Martin*, Nigel Rice** and Peter C. Smith** \\ *Department of Economics \\ **Centre for Health Economics \\ University of York
}

Corresponding author:

Peter C. Smith

Centre for Health Economics

University of York

YORK YO10 5DD

UK

Tel: 01904321443

Email: pcs1@york.ac.uk

September 2007

${ }^{1}$ This study was funded by the Health Foundation under its Quest for Quality and Improved Performance (QQuIP) initiative. We are grateful to Hugh Gravelle, Andrew Jackson and Peter Brambleby for helpful comments and advice. The material has also benefited greatly from comments from Chris Murray (Harvard Initiative for Global Health), Dean Jamison (University of California, San Francisco), participants at a seminar at the Harvard School of Public Health, and the journal's referees. 


\title{
Does health care spending improve health outcomes? Evidence from English programme budgeting data
}

\begin{abstract}
Empirical evidence has hitherto been inconclusive about the strength of the link between health care spending and health outcomes. This paper uses programme budgeting data prepared by 295 English Primary Care Trusts to model the link for two specific programmes of care: cancer and circulatory diseases. A theoretical model is developed in which decision makers must allocate a fixed budget across programmes of care so as to maximize social welfare, in the light of a health production function for each programme. This yields an expenditure equation and a health outcomes equation for each programme. These are estimated for the two programmes of care using instrumental variables methods. All the equations prove to be well specified. They suggest that the cost of a life year saved in cancer is about $£ 13,100$, and in circulation about $£ 8,000$. These results challenge the widely held view that health care has little marginal impact on health. From a policy perspective, they can help set priorities by informing resource allocation across programmes of care. They can also help health technology agencies decide whether their cost-effectiveness thresholds for accepting new technologies are set at the right level.
\end{abstract}




\section{Does health care spending improve health outcomes? Evidence from English programme budgeting data}

\section{Introduction}

One of the most fundamental yet unresolved issues in health policy is the extent to which additional health care expenditure yields patient benefits, in the form of improved health outcomes. The work of health technology agencies such as the English National Institute for Health and Clinical Excellence (NICE) has greatly improved our understanding at the micro-level of the costs and benefits of individual technologies. However, there remains a dearth of evidence at the macro-level on the benefits of increased health system expenditure.

The empirical problems of estimating the link between spending and health outcomes are manifest. If one relies on a time series of health outcome data for an individual health system it is difficult to disentangle the impact of expenditure from a wide range of other temporal influences on health, such as technological advances, epidemiological changes, and variations in broader economic circumstances. Similar methodological difficulties arise if one attempts a cross-sectional comparison of different health systems. In particular, when seeking to draw inferences from international comparisons, researchers have found it hard to adjust for all the potential external influences on health outcomes.

There is furthermore the possibility that indicators of health system inputs, such as expenditure, are endogenous, in the sense that they have to some extent been influenced by the levels of health outcome achieved in the past. And the difficulty of satisfactorily estimating the impact of health system inputs on outcomes is compounded by the great heterogeneity of health care, the multiple influences on outcomes, and the rather general nature of the outcome mortality measure traditionally used.

This paper takes advantage of a major new dataset developed in English health care, in the form of programme budgets, which enables us to address some of the 
difficulties associated with estimating the impact of health care expenditure on health outcomes. The data present expenditure on 23 broad programmes of care at the level of geographically defined local health authorities, known as Primary Care Trusts (PCTs), and embrace most items of publicly funded expenditure, including inpatient, outpatient and community care, and pharmaceutical prescriptions. They make it possible to examine the link between aggregate expenditure in a programme of care and the health outcomes achieved, notably in the form of disease specific mortality rates.

The paper models the link between spending and outcomes in two of the largest programmes of health care: circulatory disease and cancer. We start with a brief review of previous empirical studies in this domain, which have rarely yielded conclusive results. The programme budgeting data are then described, and some descriptive statistics presented. We present a simple theoretical model of the budgetary problem faced by a PCT manager seeking to allocate limited funds between competing programmes of care. Well specified econometric models are then developed that estimate (a) the budgetary expenditure choices and (b) the health outcomes achieved by PCTs in the two selected programmes of care. In contrast to many previous studies, the model results show a strong positive impact of expenditure on health outcomes. Finally, from the model results the paper is able to offer a quantitative estimate of the current cost of a life year saved in the two programmes of care. The important policy implications of these findings are discussed in the concluding section.

\section{Previous studies}

In a comprehensive review, Nolte and McKee (2004) reported many studies that seek to estimate the impact of health care and other explanatory variables on some measure of health care outcome. Usually, this production function approach employs conventional regression analysis: for example, in an early cross-sectional study of 18 developed countries, Cochrane et al (1978) used regression analysis to examine the statistical relationship between mortality rates and GNP and consumption of inputs such as health care provision. They found that the indicators of health care inputs were generally not associated with outcomes in the form of mortality rates. 
Thereafter, the failure to identify strong and consistent relationships between health care expenditure and health outcomes (after controlling for other factors) has become a consistent theme in the literature, whilst - in contrast - socioeconomic factors are often found to be highly associated with health outcomes (Nolte and McKee, 2004, p58; Young, 2001; St Leger, 2001).

However, Gravelle and Backhouse (1987) highlight the methodological difficulties associated with empirical investigation of the determinants of mortality rates. These include simultaneous equation bias and the associated endogeneity problem, and the lag between expenditure and outcomes that may occur. To avoid the difficulties imposed by data heterogeneity inherent in international analyses, the study by Cremieux et al (1999) examined the relationship between expenditure and outcomes across ten Canadian provinces over the fifteen-year period 1978-1992. They found that lower healthcare spending was associated with a significant increase in infant mortality and a decrease in life expectancy.

Although challenging the prevailing empirical orthodoxy, a difficulty with the Cremieux et al (1999) study is that the estimated regression equation consists of a mixture of potentially endogenous variables (such as the number of physicians, health spending, alcohol and tobacco consumption, expenditure on meat and fat) and exogenous variables (such as income and population density). The authors' chosen estimation technique (Generalized Least Squares) does not allow for this endogeneity and consequently the coefficients on the endogenous variables may be biased (Gravelle and Backhouse, 1987, p428). Or's (2001) study of the determinants of variations in mortality rates across 21 OECD countries between 1970 and 1995 may suffer from the same limitation. She finds that the number of doctors makes a substantial contribution to mortality reduction in OECD, but her estimation technique assumes that the number of doctors is exogenous.

Nixon and Ulmann (2006) provide a detailed review of 16 studies that have examined the relationship between health care inputs and health outcomes, using macro-level data, and undertook their own study using data for 15 EU countries over the period 1980-1995. They employed three health outcomes measures - life expectancy at birth for males and females, and the infant mortality rate - and a dozen or more 
explanatory variables including: per capita health expenditure, number of physicians (per 10,000 head of population), number of hospital beds (per 1,000 head of population), the average length of stay in hospital, the in-patient admission rate, alcohol and tobacco consumption, nutritional characteristics, and environmental pollution indicators. Nixon and Ulmann conclude that although health expenditure and the number of physicians have made a significant contribution to improvements in infant mortality, '...health care expenditure has made a relatively marginal contribution to the improvements in life expectancy in the EU countries over the period of the analysis'. Again, however, the study does not allow for the possibility that some of the explanatory variables may be endogenous.

Although loosely based on the notion of a health production function, the traditional empirical studies described above have rarely been informed by an explicit theoretical model. This is understandable, as the processes giving rise to observed health outcomes are likely to be very complex, and any theoretical model will become unwieldy. However, it leads to an atheoretical search for measures demonstrating a statistically 'significant' associations with health outcomes. In contrast, in this study we seek to inform our empirical modelling with theory. We believe that this leads to a more convincing and better specified model of health outcomes.

\section{Programme budgeting in England}

The English National Health Service (NHS) is the archetypal centrally planned and publicly funded health system. Its revenue derives almost entirely from national taxation, and access to care is generally free to the patient. Primary care is an important element of the system, and general practitioners act as gatekeepers to secondary care and pharmaceuticals. The system is organized geographically, with responsibility for the local administration of the NHS devolved to the Primary Care Trusts. For the years relevant to this study, there were 303 PCTs with average populations of 160,000 . PCTs are allocated fixed annual budgets by the national ministry, within which they are expected to meet expenditure on most aspects of health care, including inpatient, outpatient and community care, primary care and pharmaceutical prescriptions. 
Traditionally, PCTs have reported expenditure on the basis of inputs (for example, total expenditure on pay and non-pay items). However, NHS policy makers have for some time realized that this approach does not create clinically meaningful financial data or help in the design and evaluation of programmes of patient care. It therefore initiated a 'Programme Budgeting' project which has sought to create an accounting system that is more aligned with the distinct outputs and health outcomes of the health system. Since April 2003, in addition to its conventional accounting data, each PCT has prepared expenditure data disaggregated according to 23 programmes of health care. These programmes are defined by reference to the International Classification of Diseases (ICD) Version 10 codes, and most programme budget categories reflect ICD 10 chapter headings (e.g., cancer and tumours, circulation problems, renal problems, neonates, problems associated with the skin, vision, hearing, etc). In some cases, the 23 categories are broken down into further sub-areas. For example, the large mental health category is broken down into 'substance abuse', 'dementia', and 'other'. The reported expenditure relates to all public expenditure on residents of a PCT, regardless of where the provider is located.

Programme budgeting seeks to allocate all types of PCT expenditure to the various programme budget categories, including secondary care, community care and prescribing. However, the system acknowledges that a medical model of care may not always be appropriate, and two specific non-clinical groups - 'Healthy Individuals' and 'Social Care Needs' - have been created. These are intended to capture the costs of disease prevention programmes and the costs of services that support individuals with social rather than health care needs. In addition, it is in some cases not possible to assign activity by medical condition, preventative activity, or social care need, in which case expenditure is assigned to a category entitled 'Other'. The use of this category ensures all expenditure can be assigned to a programme of care (Department of Health, 2005a, p7).

The aim of the programme budget classifications is to identify the entire volume of health care resources assigned to broad areas of illness according to the primary diagnosis associated with an intervention. It serves a number of purposes, most notably to assist in the local planning of health care. But for this study its crucial merit is that it opens up the possibility of examining the statistical relationship between 
local programme spending and associated disease-specific outcomes.

Programme budgeting information was first collected in financial year 2003/04, and in this paper we report information for the second year of implementation, FY 2004/05. The first column of Table 1 shows the national average NHS expenditure per person by programme budget category. Across England as a whole, NHS expenditure per person is $£ 1,183$. The single largest category is the 'other' category (category 23) with expenditure per person of almost $£ 155$ in 2004/05. By far the largest element within this category is primary care expenditure, which accounts for $£ 127$ per head.

$<$ Table 1 about here $>$

There are two other categories with an expenditure level over $£ 100$ per head: mental health problems (category 5) attract an annual expenditure of $£ 145$ per person, and circulation problems (category 10) receive $£ 122$ per person. Next come four programme budget categories - cancers and tumours, gastro-intestinal problems, musculo-skeletal problems, and trauma, burns and injuries - with an annual expenditure of between $£ 71$ and $£ 75$ per person. Respiratory and genito-urinary problems both record an expenditure of $£ 62$ per person, with maternity and reproductive conditions being allocated $£ 55$ per head. There is no evidence of major shifts in the data from FY 2003/04, suggesting the data are reasonably stable.

The remainder of Table 1 indicates the variation in expenditure amongst PCTs. For each programme budget category, the PCT's per capita expenditure is adjusted for unavoidable geographical variation in costs. This is adjustment is necessary because input prices in London and the south east of England are up to 30\% higher than elsewhere. The cost adjustment is achieved by adjusting raw figures according to a price index reflecting input costs in the locality (the Hospital and Community Health Services Market Forces Factor: Department of Health, 2005b). ${ }^{2}$ The unweighted

\footnotetext{
${ }^{2}$ An MFF is not available for prescribing expenditures. To test how sensitive our results are to the exclusion of a prescribing adjustment we calculated an MFF which, for each PCT, is a weighted average of the national average HCHS MFF and the PCT's own HCHS MFF with weights reflecting the national proportion of expenditure on drugs (p) and the national proportion of expenditure on all other items (1-p) for the expenditure category concerned (e.g. for cancer, for circulation problems, and
} 
average of these PCT expenditure per capita figures is reported for each programme budget category in the second column of Table 1, followed by the observed minimum and maximum. The final column shows the coefficient of variation.

The variation in expenditure levels across PCTs is considerable. For example, expenditure per head on cancers and tumours averages $£ 76$ across all PCTs but this varies between $£ 39$ and over $£ 133$ per head (see also Figure 1 which provides a plot of per capita PCT expenditure on cancer). Similarly, expenditure per head on circulation problems averages $£ 124$ across all PCTs but this varies between $£ 64$ and over $£ 186$ per head (see also Figure 2 which provides a plot of per capita PCT expenditure on circulation problems). Although there is clearly variation within these two particular programme budget categories, this variation is relatively small compared to other programme categories - such as infectious diseases and blood disorders - that have much larger coefficients of variation.

$<$ Figures 1 and 2 about here $>$

A fundamental analytic difficulty is that of disentangling the impact of medical needs from expenditure on health outcomes. To illustrate, Figure 3 plots total PCT per capita expenditure (adjusted for local cost conditions) against the PCT mortality rate for those aged under 75 for deaths from all causes amenable to health care from 2002 to 2004 (see Table A1 in the Appendix for details of the causes defined as 'amenable to health care'). It reveals a clear positive relationship between these two variables (correlation coefficient 0.624 ). A similar but weaker positive relationship (not shown here) also exists between PCT per capita expenditure on cancer services and the cancer SMR for those aged under 75 (correlation coefficient 0.213). The relationship between PCT per capita expenditure on circulatory problems and the under-75 circulatory disease SMR has a correlation coefficient of 0.304 .

$<$ Figure 3 about here $>$

Thus, as is frequently the case, the programme budgeting data indicate a strong so on). Re-calculation of the per-capita expenditure data to incorporate this MFF adjustment left the 
positive relationship between health care spending and adverse outcomes, apparently contradicting the hypothesis that PCTs that spend more on health care will achieve better health outcomes However, interpretation of this finding is not straightforward, as much of the variation in expenditure across PCTs will reflect different levels of the need for health care. Areas with a relatively large proportion of elderly residents, or operating in relatively deprived locations, can be expected to experience relatively high levels of spending. Adjusting for the relative health care needs of different populations is therefore a central requirement of any analytic effort in this domain.

Fortunately the Department of Health has a well-developed methodology for estimating the relative health care needs of PCTs, in the form of the weighted capitation formula it uses as the basis for allocating health care funds to PCTs (Smith, Rice and Carr-Hill, 2001). The current 'needs' formula is derived from an adjustment for the demographic profile of the PCT and a series of econometric analyses of the link between health care expenditure and other socio-economic factors at a small area level within England (Department of Health, 2005b).

The plot in Figure 4 is similar to that in Figure 3 but holds constant the local need for health care (by dividing expenditure by the index of needs used by the Department of Health). It therefore plots total expenditure per capita (adjusted for local cost and need conditions) against the SMR for those aged under 75 from all causes of death amenable to health care. The positive association between expenditure and deaths observed in Figure 3 is now dramatically reversed. That is, it suggests that - once the need for health care is held constant - more expenditure is associated with a better outcome (a lower death rate). Similar results obtain when allowance is made for local cost and need conditions in specific programmes of care. The correlation coefficient between all expenditure and the mortality rate for deaths amenable to health care becomes -0.451 . For cancers and tumours it is -0.323 , and for circulation problems it is -0.358 .

$<$ Figure 4 about here $>$

results largely unchanged. 
The purpose of this paper is to integrate the rudimentary findings illustrated in this section into a coherent model of expenditure and outcomes, and to estimate the strength of the relationships suggested in the preceding paragraphs. To provide an analytic framework, the next section presents a theoretical model of PCT expenditure allocation across the 23 programme budgeting categories.

\section{Theoretical model}

We assume each PCT receives an annual financial lump sum budget $y_{i}$ from the national ministry, and that total expenditure cannot exceed this amount. The PCT must then decide how to allocate the expenditure across $J$ programmes of care $(j=1, \ldots, J ; J=23$ in this case). For the $j$-th programme of care there is a 'health production function' $f_{j}($.$) that indicates the link between local spending x_{i j}$ on programme $j$ and health outcomes in that programme, $h_{i j}$. Health outcomes might be measured in a variety of ways, but the most obvious is to consider some measure of improvement in life expectancy, possibly adjusted for quality of life, in the form of a quality adjusted life year.

The nature of the specific health production function confronted by a PCT will depend on two types of local factors: the clinical needs of the local population relevant to the programme of care (which we denote $\boldsymbol{n}_{\boldsymbol{i j}}$ ) and broader local environmental factors $\mathbf{z}_{\boldsymbol{i j}}$ relevant to delivering the programme of care (such as input prices, geographical factors, or other uncontrollable influences on the production function). Both clinical and environmental factors are in general multidimensional in nature, hence the vector representation. Increased expenditure then yields improvements in health outcomes, as expressed for example in improved local mortality rates, but at a diminishing rate. That is:

$$
h_{i j}=f_{j}\left(x_{i j}, \mathbf{n}_{i j}, \mathbf{z}_{i j}\right) ; \partial f_{j} / \partial x>0 ; \partial^{2} f_{j} / \partial x^{2}<0
$$

We assume there is a PCT social welfare function $W($.) that embodies health outcomes across the $J$ programmes of care. Assuming no interaction between programmes of care, each PCT $i$ allocates its total budget $y_{i}$ so as to maximise total welfare subject to 
local budget constraint and the health production functions for each programme of care:

$$
\begin{array}{ll}
\max & W\left(h_{i 1}, h_{i 2}, \ldots, h_{i J}\right) \\
\text { subject to } & \sum_{j} x_{i j} \leq y_{i} \\
& h_{i j}=f_{j}\left(x_{i j}, \mathbf{n}_{i j}, \mathbf{z}_{i j}\right) ; \quad \mathrm{j}=1, \ldots \mathrm{J}
\end{array}
$$

It can of course quite plausibly be argued that decision-makers do not discriminate between health outcomes in different programmes of care, and that $\mathrm{W}($.$) is merely the$ sum of such outcomes. However, there is no need for that assumption in our formulation.

This model implies that a PCT allocates its budget across the 23 programmes of care so that the marginal benefit of the last pound spent in each programme of care is the same. Solving the constrained maximisation problem yields the result that the optimal level of expenditure in each category, $x_{i j}{ }^{*}$, is a function of the need for health care in each category ( $\left.\boldsymbol{n}_{\boldsymbol{i 1}}, \boldsymbol{n}_{\mathbf{i 2}}, \ldots, \boldsymbol{n}_{\boldsymbol{i k}}\right)$, environmental variables affecting the production of health outcomes in each category $\left(\mathbf{z}_{\mathbf{i} 1}, \mathbf{z}_{\mathbf{i} 2}, \ldots, \mathbf{z}_{\boldsymbol{i k}}\right)$, and PCT budget $\left(y_{i}\right)$.

$$
x_{i j}^{*}=g_{j}\left(n_{i 1}, \ldots n_{i k}, z_{i 1}, \ldots z_{i k}, y_{i}\right) ; \quad \mathrm{i}=1, \ldots, \mathrm{m} ; \mathrm{j}=1, \ldots, \mathrm{k}
$$

Thus, for each programme of care there exists an expenditure equation (3) explaining expenditure choice of PCTs and a health outcome equation (1) that models the associated health outcomes achieved. This paper seeks to estimate these equations empirically for two programmes of care.

Our model is static, in the sense that the health production function (2) assumes that all health benefits occur contemporaneously with expenditure. We acknowledge that for some programmes of care benefits might occur one or more years after expenditure has occurred. This is particularly likely to be the case for those programmes aimed at encouraging healthy lifestyles, where some benefits may occur decades after the actual programme expenditure. For other programmes, such as maternity/reproductive conditions and neonate conditions, benefits may be largely contemporaneous with expenditure. Furthermore, we do not model the decision 
maker's time preferences. For our empirical modelling, however, we are constrained by the data we have available, which are largely cross-sectional in nature. Implicitly we have to assume that the data represent a quasi long-run equilibrium position, and that relative expenditure levels and health outcomes within each PCT have been reasonably stable over a period of time. In the English context, this appears to be a reasonable assumption, although as panel data become available it will be come possible to develop a more dynamic model.

\section{Model estimation}

The theoretical model suggests the specification and estimation of a system of equations, with an expenditure and health outcome equation for each of the 23 programmes of care. In the absence of endogenous regressors the system would reduce to the estimation of seemingly related regressions. However, this approach makes infeasible data demands, requiring variables to identify expenditure, need, environmental factors and health outcomes in each of the 23 programmes of care. In the presence of endogenous expenditure and outcome data, the approach would further require a set of exogenous variables to act as instruments to identify the system.

At the time of writing the Department of Health has made available health outcome indicators for only two disease categories: cancer and circulatory problems. Further, we do not have convincing data on all the environmental factors likely to affect the production of health care. As a result, we concentrate on modelling these two large programmes of care separately. In line with our theoretical model, for each programme we specify the following reduced forms for models of expenditure (4) and health outcome (5):

$$
\begin{aligned}
& x_{i l}=\alpha_{1}+\beta_{1} n_{i l}+\lambda y_{i}+\varepsilon_{1 i l} \quad i=1, \ldots, m ; \quad l=1, \ldots, 2 . \\
& h_{i l}=\alpha_{2}+\beta_{2} n_{i l}+\delta x_{i l}+\varepsilon_{2 i l}
\end{aligned}
$$

Ideally we should use prevalence data as a basis for modelling clinical needs in the programmes of care. These are not available. We therefore proxy health care need in 
each of the cancer and circulatory disease models using the 'needs' component of the resource allocation formulae. The needs element of the Department of Health formula was specifically designed to adjust PCT allocations for local health care needs and accordingly, ceteris paribus, we would expect a positive relationship between expenditure $x_{i l}$ and need $n_{i l}$ for the two programmes of care. We would also expect a positive relationship between need and adverse health outcomes $h_{i l}$.

For each programme of care, we develop models using two alternative measures of health outcomes: the disease-specific standardized mortality ratio for those aged under 75, and a measure of the avoidable years of life lost (YLL) to the disease. The latter variable is calculated by summing over ages 1 to 74 years the number of deaths at each age multiplied by the number of years of life remaining up to age 75 years. The crude YLL rate is simply the number of years of life lost divided by the resident population aged under 75 years. Like conventional mortality rates, the YLL rate can be age-standardised to eliminate the effects of differences in population age structures between areas, and this is the variable employed in our study (Lakhani et al., 2006 p379)

The expenditure equations to be estimated also require us to use a proxy for needs across the other programmes of care. We therefore model need for competing programmes by the standardised mortality data in the other programme of care (this is of course treated as an endogenous variable). That is, for circulatory disease expenditure, we use the all age standardised mortality rate for cancer, and likewise for cancer expenditure, the all age standardised mortality rate for circulatory diseases is applied.

Our estimation strategy is as follows. First we estimate the reduced form models using OLS. Assuming exogeneity of health outcomes in the expenditure model (4), and of expenditure in the health outcome model (5) OLS is a consistent estimator of the model parameters. However, should these variables be endogenous, then we violate one of the assumptions of least squares as the endogenous variables will be correlated with the disturbance term in their respective model. We test for endogeneity using the test proposed by Durbin (1954). Under the null hypothesis of 
exogeneity, OLS will yield consistent parameter estimates. The test consists of comparing OLS estimates to those produced by instrumental variables estimators such as two-stage least squares (2SLS). A large discrepancy between the estimates indicates a rejection of exogeneity. Under the null, the test statistic is distributed as chi-squared with degrees of freedom equivalent to the number of regressors deemed endogenous.

We have a number of potential instruments available, mostly derived from 2001 Population Census. ${ }^{3}$ Of these we attempt to select appropriate instruments on both technical and pragmatic grounds. From a pragmatic point of view, we require a parsimonious set of instruments that satisfy the necessary technical criteria. These are, firstly, that they have face validity, that is, that they are plausible determinants of the endogenous variable being instrumented, and secondly, that the instruments are both relevant and valid. The relevance of an instrument set refers to its ability to predict the endogenous variable of concern, whereas validity refers to the requirement that instruments should be uncorrelated with the error term in the equation of interest.

If there is evidence of endogeneity of expenditure and health outcomes we implement two-stage least squares. Should the instrument set be relevant and valid, two-stage least squares will produce consistent estimates of the parameters of the reduced form models. We subject the instrument sets to tests for validity using the Sargan (1958) test of overidentifying restrictions. Under the null hypothesis that the instruments are uncorrelated with the disturbance and are correctly excluded from the equation of interest, the test statistic is distributed as chi-squared in the number of overidentifying restrictions.

In addition to the Sargan test, we test for instrument relevance using the Anderson (1984) canonical correlations likelihood-ratio test. If the instrument set is considered weak (marginally relevant) this may lead to biased two-stage least squares estimates of our equation of interest. The likelihood ratio test of Anderson (1984) is a test of whether the equation is identified and under the null that the equation of interest is underidentified, the Anderson statistic is distributed as chi-squared with degrees of

\footnotetext{
${ }^{3}$ In total, 22 variables could be considered potential instruments.
} 
freedom equal to $(l-k+1)$, where $l$ is the number of instruments (included and excluded exogenous variables) and $k$ is the total number of regressors. Rejection of the null, indicates that the model is identified.

While the Anderson statistic provides a test of the null hypothesis of unidentification, Stock and Yogo (2002) suggest a test for the null that the instruments are weak and provide appropriate critical values. The test is an extension of the Cragg-Donald (1993) test for identification. In the presence of a single endogenous regressor the statistic is based on the F statistic for testing the null hypothesis that the instruments do not enter the first stage regression of two-stage least squares. A general test of model specification is provided through the use of Ramsey's (1969) regression error specification test for OLS and an adapted version of the test for instrumental variables (Pesaran and Taylor, 1999). This test operates under the null hypothesis that there are no neglected nonlinearities in the functional form of the model specified. The standard Reset test implemented using OLS estimation follows an F distribution while the two-stage least squares equivalent follows a chi-square distribution. Both have degrees of freedom equal to the number of polynomial terms chosen for the fitted values. We implement the test using $\hat{y}^{2}, \hat{y}^{3}$ and $\hat{y}^{4}$, with three degrees of freedom.

\section{Empirical results}

Eight of the 303 PCTs were excluded from the analysis because they were subject to boundary changes in the year studied, leading to incomplete data. The analysis therefore uses 295 observations throughout. We first present results for the cancer programme of care in Table 2. Columns under (1) present the OLS results for the two equations to be estimated using SMRs as the death measure and columns under (2) present comparable two-stage least squares results. Columns under (3) and (4) employ standardised years of life lost rates (SYLLs) rather than SMRs as the death indicator with (3) presenting two-stage least squares results and (4) containing comparable OLS results. All variables have been log transformed and accordingly parameter estimates can be interpreted as elasticities.

$<$ Table 2 about here $>$ 
Consider first the OLS results under (1). These indicate that both the cancer death rate and cancer expenditure are positively correlated with need. However, while the results suggest that expenditure on cancer services is negatively related to cancer deaths, the effect is very small and fails to achieve significance. Expenditure on cancer is also negatively related to other calls on expenditure - the non-cancer death rate - as proxied here by the circulation death rate. The estimated coefficient suggest that a $10 \%$ increase in calls on other expenditures results in a $3.8 \%$ reduction in cancer expenditure. The OLS results under (4), which replace mortality rates with years of life lost rates, are very similar.

The second set of results (under (2)) present two-stage least squares estimates using SMRs as the outcome indicator. Our instrument set consists of the proportion of households that are lone pensioner households and the proportion of the population providing unpaid care. These instruments have intuitive appeal. The first stage regression of cancer expenditure on the instruments and the need for health care (as an exogenous regressor in the 2SLS model) reveals a positive and significant coefficient on lone pensioners and a negative but non-significant coefficient on the proportion of unpaid carers (see Table A2 in the appendix). The proportion of lone pensioners is likely to reflect an additional adjustment for health care need specific to an elderly and needy population. Unpaid care is a substitute for the provision of health care services and accordingly one may expect a negative relationship with expenditure. Similarly for the cancer expenditure model, the first stage regression of the instrument set (including need and total budget) on circulatory deaths results in a negative coefficient on both instruments. A greater proportion of unpaid carers reflects an increased level of care (and perhaps increased compliance with care programmes and drug regimes) resulting in a decrease in circulatory deaths. Conditional on need and the total PCT budget, the negative coefficient on the proportion of lone pensioners may be indicative of areas with increased networks of social support, or reflect a selection effect, in the sense that areas with a low under 75 death rate may as a result have an older age structure.

The 2SLS results under (2) suggest that both cancer deaths and expenditure are more 
elastic with respect to health needs compared to the OLS results under (1). We further observe a large and positive relationship between total PCT budget and expenditure on cancer services, suggesting that a $10 \%$ increase in budget leads to a 8.7\% increase in cancer expenditure. This implies that increases in budget may be distributed across programme budgets roughly in proportion to existing allocations, a rational finding that lends face validity to the model specifications. Expenditure is also highly responsive to need for non-cancer care (an elasticity of -0.576).

The main difference between the OLS and 2SLS results is the increased negative coefficient on cancer expenditure in cancer deaths equation. This change is to be expected as 2SLS treats expenditure as endogenous to health outcomes. The 2SLS results indicate that a $10 \%$ increase in cancer programme expenditure results in approximately a $4.9 \%$ reduction in adverse health outcomes, observed through cancer deaths.

There is clear evidence that the OLS model under (1) is misspecified $(F(3,289)=11.29 ; p=.000)$, and it should therefore be rejected in favour of the 2SLS model. Further support for the 2SLS model is provided through the Sargan test of overidentifying restrictions, the Anderson and Cragg-Donald tests of instrument relevance and the partial R-squared values from the first stage regressions of the set of exogenous variables on the relevant endogenous variable. These tests indicate that the instrument set is both valid and relevant. Further, the assumption of exogeneity of deaths or expenditure can be rejected in all models.

Substituting years of life lost rates for mortality rates generates qualitatively similar 2SLS results (the 2SLS SYLL results are shown under (3)). Moreover, the use of the SYLL variable allows us to estimate the implicit cost of a life year 'saved' in cancer services. The estimates suggest a $1 \%$ increase in cancer expenditure per head - which was $£ 75.1$ in $2004 / 5$ - gives rise ceteris paribus to a $0.378 \%$ reduction in years of life lost. Across 2002-04, total life years lost to cancer deaths in those aged under 75 was 2,268,541 (or 756,180 life years per annum). Across the English population of 50 million, this suggests 0.015 life years (5.52 days) per person. Thus a $1 \%$ increase in expenditure per head (£0.751) is associated with a $0.378 \%$ reduction in life years lost 
(0.021 days) and implies that one life year would cost $£ 13,137$. Furthermore, using the estimated standard errors from the model, we calculate the 95\% confidence interval surrounding this estimate to be $£ 9,118$ to $£ 23,490{ }^{4}$

Results for circulatory diseases are shown in Table 3. An important additional consideration in the modelling of circulatory disease was the failure of initial 2SLS models to pass the Sargan test for instrument validity in model (2) and the general specification tests when using years of life lost in model (3). Careful scrutiny of these results indicated that they arose from the failure to model expenditure satisfactorily in a small number of PCTs with high levels of non-white residents. The expenditure models were therefore re-estimated with an additional 'needs' variable, in the form of the percentage of the population in a 'white' ethnic group. This variable exhibited strong positive association with expenditure, other things equal, and its introduction led to a well-specified model. ${ }^{5} 6$ In common with models for cancer, all circulatory models now appear well specified with valid and relevant instruments.

$<$ Table 3 about here $>$

The two instruments used for cancer are used here augmented with the population weighted index of multiple deprivation (IMD 2000). This is plausible, as circulatory disease is more related to disadvantage than cancer. Relationships between the instruments and the endogenous regressors similar to those found for cancer are observed here (see Table A3 in the appendix for the first-stage regressions). The

\footnotetext{
${ }^{4}$ Lichtenberg (2004) estimated the marginal drug cost associated with an extra life year for someone diagnosed with cancer. He employed US panel data to estimate the relationship between drug expenditure and pharmaceutical vintages on cancer survival rates. Lichtenberg found that cancers for which the stock of drugs increased more rapidly tended to have greater increases in survival rates. New cancer drugs increased the life expectancy of people diagnosed with cancer by about one year from 1975 to 1995 and the estimated drug cost to achieve the additional year of life per person diagnosed with cancer was below $\$ 3000$ at 1995 prices (Lichtenberg, 2004).

${ }^{5}$ Running the model without the ethnicity variable resulted in the following coefficients on the circulatory expenditure model: constant 8.834 (1.872); need 1.597 (.245); total budget 692 (.156); noncirculatory deaths -1.858 (.290). Because of potential problems of misspecification we have greater confidence in the results reported in Table 3 which include the percentage of the population in a 'white' ethnic group.

${ }^{6}$ The positive relationship between the percentage of the population from 'white' ethnic groups and circulatory disease expenditures may well reflect concerns about reduced access to health care services among ethnic minority groups in the UK (Gill et al., In Press).
} 
higher the proportion of pensioners living alone and unpaid carers, the lower the under 75 years cancer death rate, while greater deprivation is associated with increased death rates. Increased expenditure on circulatory disease in the first stage regressions is associated with a greater proportion of pensioners living alone and a greater proportion of unpaid carers. The latter may reflect an increased awareness and compliance with medical intervention, particularly preventative measures, brought about by carers.

In general, the set of estimated coefficients for circulatory problems are more elastic than their cancer counterparts. For example, a 10\% increase in health need results in an increase in circulatory expenditure of between 5.8\% (OLS using SYLL measures) and $12.8 \%$ (2SLS using SYLL measures). As we move from OLS to 2SLS we observe an increase in the absolute value of the estimated coefficients attached to the endogenous regressors. In particular, there is more than a three-fold increase in the estimated coefficient on circulatory expenditure. Further, the 2SLS coefficients of 1.387 and -1.427 imply that circulatory disease outcomes are more responsive to increases in expenditure than their cancer counterparts. Using either outcome measure, a $10 \%$ increase in expenditure is associated with a $14 \%$ reduction in the death rate. The coefficient on the non-circulatory mortality rate in the circulatory expenditure model also increases, from -0.400 for OLS to -1.052 for 2SLS.

The results of the 2SLS circulatory expenditure model for years of life lost can be used in an analogous manner to those for cancer to calculate the marginal cost of a life year lost. The circulatory expenditure coefficient of -1.427 implies that a $1 \%$ increase in expenditure gives rise to a $1.4 \%$ reduction in life years lost to circulatory disease. Across 2002-04, life years lost to all circulation deaths in those aged under 75 was 1,607,171, or 535,724 life years per annum. Across an English population of 50 million, this suggests 0.0107144 life years (3.91 days) per person. Thus a $1 \%$ increase in expenditure per head $(£ 1.22)$ is associated with a $1.4 \%$ reduction in life years lost (0.056 days) and implies that one life year would cost $£ 7,979$. Using the estimated standard errors suggests that the 95\% confidence interval surrounding this estimate is considerably smaller than for cancer (£6,549 to $£ 10,208)$.

Our results are presented in terms of unadjusted life years. In order to give a very 
rough indication how they might be adjusted to yield quality-adjusted life years

(QALYs), we have applied the utility scores made available by the HODaR project

(HODaR, University Hospital of Wales) using the UK EQ-5D scoring algorithm.

Quality of life scores are available for by ICD10 codes and can be assigned to the

programme budget categories used here. We have therefore simply assigned scores to

each of the ICD10 categories with the programme budgeting areas of cancer and circulatory diseases where these match with the HODaR categories, and averaged the scores across the categories ${ }^{7}$. Using this method, for cancer expenditure cost of a

QALY is $£ 19,070$, whilst for circulatory diseases the corresponding figure is $£ 11,960$.

We emphasize that these results are at best indicative and cannot offer an accurate calculation of a quality-adjusted life year saved, but they do suggest that the cost of a QALY from these programmes of care may be lower than many commentators have assumed. $^{8}$

\footnotetext{
${ }^{7}$ Utility scores are available for ICD10 codes based on EQ-5D (HODaR). These are derived from a sample of 15,113 subjects accounting for more than 37,000 ICD10 observations (due to multiple diagnoses). Averaging utility scores across the ICD10 codes corresponding to the cancer programme of care (note that not all ICD10 codes corresponding to the cancer programme of care were represented in the HODaR sample) resulted in an average score of .689. The corresponding calculation for circulatory diseases is .669. Note that these are very rough estimates. To accurately calculate the cost of a qualityadjusted life year saved we would require utility scores for all of the programme budgeting ICD10 codes together with the number of patients assigned to each of these codes. We do not have full information on these. It is also noted that the utility scores may be based on small samples (five or more subjects). The utility scores were made available by Dr Craig Currie, Director and Senior Lecturer in Health Outcomes Research, HODaR, Cardiff Medicentre, University Hospital of Wales.

${ }^{8}$ We are grateful to an anonymous referee for pointing out that these cost per QALY estimates may be biased upwards because they do not include the benefits to patients who receive treatment for but do not die from cancer or circulatory disease. Such treatment may improve the quality of life of those with these diseases but, if the treatment does not affect their mortality, its benefits will not be captured in our QALY adjusted cost of life estimates. The denominator in the cost of a life year saved calculation incorporates only deaths deferred within the relevant programme, and the associated life years gained are then quality adjusted using the HODaR data. Consequently, to the extent that they do not account for all of the benefits from the expenditure associated with a programme, the QALY adjusted cost of a life year saved estimates may be biased upwards.
} 


\section{Conclusions}

This study shows that health care expenditure has a strong positive effect on outcomes in the two programmes of care investigated. Our estimates suggest that, relative to received wisdom, the marginal cost of a 'life year' saved is quite low, at approximately $£ 8,000$ for circulatory disease and $£ 13,100$ for cancer. ${ }^{9}$ Incorporation of a rudimentary quality adjustment suggests that the corresponding costs of a QALY saved are respectively $£ 11,960$ and $£ 19,020$. These estimates are associated with quite large confidence intervals (especially for cancer), but they do appear to compare quite favourably with the sum of $£ 30,000$ for a quality-adjusted life year commonly attribute to NICE as the threshold for accepting new technologies.

There is clear evidence that expenditure on circulatory disease yields greater benefits in terms of life years than expenditure on cancer. This is to be expected. Recent developments in circulatory drug therapies (especially statins) are acknowledged to be highly cost-effective. Furthermore, a substantial element of cancer expenditure is devoted to palliative care, the benefits of which are unlikely to be measured to any great extent in increased life expectancy.

The models offer evidence of a strong substitution effect between expenditure on programmes of care. Other things being equal, expenditure on a specific programme is depressed in the face of higher need in other programme areas. Our results suggest that PCTs appear to be acting rationally by directing their budget to the programme areas that will yield greatest health benefit for their locality.

A by-product of the modelling has been the discovery of strong evidence of either lower levels of need or 'unmet' need amongst the non-white population in circulatory disease. This is signalled by the need to incorporate the 'white' ethnic group variable into the circulatory expenditure models. Further analysis of this finding is beyond the remit of this study, given the limited data available to us. However, the strength of the

\footnotetext{
${ }^{9}$ Since the paper was written, data for the financial year 2005/06 have been released. They yield models that are very similar both qualitatively and quantitatively, suggesting that the results obtained are robust across years.
} 
effect leads us to recommend that policy makers in England should examine with some urgency whether circulatory expenditure on 'non-white' ethnic groups is below that on their 'white' counterparts, after adjusting for clinical need.

The dramatic change in inference that arises from moving from the misspecified OLS models to the well-specified 2SLS models illustrates why proper econometric modelling is needed if the relationship between expenditure and health outcome is to be investigated correctly. The models and methods described here are essential if incorrect inferences are to be avoided. In particular, they suggest a far more marked influence of health care spending on health outcomes than is often indicated by more conventional analysis.

We nevertheless recognize that this study has a number of limitations. It uses limited health outcomes data (in the form of mortality data for just two programmes of care), and we would hope that in time a greater range of outcome and epidemiological data will be made available in the future. Furthermore, we have modeled just a single year's data. In practice health outcomes are the results of years of expenditure by local PCTs, and conversely current expenditure is expected to yield outcome benefits beyond the current year. Implicitly, our analysis assumes that PCTs have reached some sort of equilibrium in the expenditure choices they make and the outcomes they secure. This is probably not an unreasonable assumption, given the relatively slow pace at which both types of variable change. But a longer time series of data may enable us to model the effects with more confidence.

More generally, the English programme budgeting project is a major new data development. However, it is still under development, and there remain unresolved issues. Some health system expenditure is difficult to assign to programmes, most notably in primary care . Furthermore, accounting practice is variable, and we would recommend that programme budgeting accounts should in future be properly audited.

We nevertheless believe that programme budgeting is a major initiative that should be actively and vigorously promoted by other health systems. Most importantly, it brings together for the first time clinical data (in the form of health outcomes) and expenditure data. It therefore permits researchers to model links between health care 
expenditure and health outcomes in a much more secure manner than hitherto. It enables policy makers to make better informed decisions about where their limited budgets are best spent. And it can also help health technology assessment agencies determine whether their current cost-effectiveness threshold for accepting new technologies is set at an appropriate level. 


\section{References}

Anderson, T.W. 1984. Introduction to multivariate statistical analysis. $2^{\text {nd }}$ Ed. New York, John Wiley \& Sons.

Cochrane, A., St Leger, A.S, and Moore, F. (1978). Health service 'input' and mortality 'output' in developed countries. Journal of Epidemiology and Community Health, 32, 200-205.

Cragg, J.G., Donald, S.G. (1993). Testing identifiability and specification in instrumental variables models. Econometric Theory; vol9: 222-240.

Cremieux, P, Ouellette, P and Pilon, C (1999). Health care spending as determinants of health outcomes. Health Economics, 8, 627-639.

Department of Health (2005a). NHS Finance Manual. December 2005 edition. See http://www.dh.gov.uk/assetRoot/04/13/18/26/04131826.pdf

Department of Health (2005b). Unified exposition book: 2003/04, 2004/05 and 2005/06 PCT revenue resource limits. Department of Health, London.

Durbin, J. 1954. Errors in variables. Review of the International Statistical Institute. Vol 22; 23-32.

Gill, P.S., Kai, J., Bhopal, R.S., Wild, S. Health care needs assessment: black and minority ethnic groups. In: Stevens, Raftery, Mant, and Simpson (eds.). Health Care Needs Assessment. The Epidemiologically Based Needs Assessment Reviews, Third Series. Abington: Radcliffe Medical Press Ltd. (in press).

Gravelle, $\mathrm{H}$ and Backhouse, M (1987). International cross-section analysis of the determination of mortality. Social Science Medicine, 25, 5, 427-441.

Lichtenberg, F. (2004). The expanding pharmaceutical arsenal in the war on cancer. NBER working paper no. 10328, February.

Nixon, J. and Ulmann, P (2006). The relationship between health care expenditure and health outcomes. European Journal of Health Economics, 7, 7-18.

Nolte, E and McKee, M (2004). Does health care save lives? The Nuffield Trust, London. 
Or, Z (2001). Exploring the effects of health care on mortality across OECD countries. OECD Labour Market and Social Policy Occasional Paper No 46. OECD, Paris.

Pesaran, M.H. Taylor, L.W. 1999. diagnostics for IV regressions. Oxford Bulletin of Economics and Statistics, Vol 61, No 2: 255-81.

Ramsey, J.B. 1969. Tests for specification errors in a classical linear least squares regression analysis. Journal of the Royal Statistical Society, Series B; Vol 31: 350-71.

Sargan, J.D. 1958. The estimation of economic relationships using instrumental variables. Econometrica, 26: 393-415.

Smith, P.C., Rice, N., Carr-Hill, R.A. (2001). Capitation funding in the Public Sector. Journal of the Royal Statistical Society, Series A, 164 Part 2: 217-257.

St Leger, S (2001). The anomaly that finally went away. Journal of Epidemiology and Community Health, 55, 79.

Stock, J.H., Yogo, M. 2002. Testing for weak instruments in linear IV regression. NBER Technical Working Paper 284.

Young, F W (2001). An explanation of the persistent doctor-mortality association. Journal of Epidemiology and Community Health, 55, 80-84. 
Table 1 Descriptive statistics by programme budget category per person all England, using cost adjusted expenditure by PCT, FY2004-05

\begin{tabular}{|c|c|c|c|c|c|c|}
\hline \multirow{2}{*}{\multicolumn{2}{|c|}{$\begin{array}{l}\text { Programme budget } \\
\text { category }\end{array}$}} & $\begin{array}{l}\text { National } \\
\text { net spend }\end{array}$ & \multicolumn{4}{|c|}{ PCT spend per head $£$, cost adjusted } \\
\hline & & $\begin{array}{l}\text { per head, } \\
£, 2004-05\end{array}$ & Mean & Minimum & Maximum & $\mathrm{CV}$ \\
\hline 1 & Infectious diseases & 20.1 & 18.6 & 8.9 & 137.6 & 0.68 \\
\hline 2 & Cancers/tumours & 75.1 & 75.8 & 39.1 & 133.4 & 0.21 \\
\hline 3 & Blood disorders & 16.9 & 16.4 & 3.8 & 58.1 & 0.46 \\
\hline \multirow[t]{3}{*}{4} & Endocrine/metabolic & 31.7 & 31.7 & 12.4 & 51.5 & 0.18 \\
\hline & Diabetes & 13.5 & 13.4 & 0.0 & 33.3 & 0.34 \\
\hline & Other & 18.2 & 18.2 & 0.0 & 40.9 & 0.30 \\
\hline \multirow[t]{4}{*}{5} & Mental health & 145.3 & 142.9 & 51.2 & 323.3 & 0.28 \\
\hline & Substance abuse & 11.9 & 12.2 & -2.0 & 146.8 & 1.37 \\
\hline & Dementia & 16.1 & 16.3 & 0.0 & 158.3 & 1.28 \\
\hline & Other & 117.3 & 114.3 & 0.0 & 247.8 & 0.34 \\
\hline 6 & Learning disability & 42.0 & 42.5 & 4.7 & 163.3 & 0.46 \\
\hline 7 & Neurological system & 34.9 & 35.5 & 18.6 & 70.6 & 0.24 \\
\hline & Eye and vision & 27.5 & 28.2 & 4.5 & 65.7 & 0.30 \\
\hline & Hearing & 6.3 & 6.3 & 1.7 & 32.7 & 0.47 \\
\hline & Circulation (CHD) & 122.0 & 124.1 & 64.0 & 186.3 & 0.19 \\
\hline & Respiratory & 62.5 & 63.7 & 30.3 & 147.6 & 0.25 \\
\hline & Dental & 13.3 & 13.4 & 0.0 & 96.4 & 0.80 \\
\hline 13 & Gastro Intestinal & 73.0 & 74.4 & 34.4 & 132.3 & 0.22 \\
\hline 14 & Skin & 24.8 & 24.9 & 13.2 & 49.7 & 0.27 \\
\hline & Musculo Skeletal & 71.2 & 72.3 & 19.1 & 157.6 & 0.23 \\
\hline & Trauma/injuries & 71.9 & 72.7 & 35.2 & 209.1 & 0.26 \\
\hline 17 & Genito/urinary & 62.1 & 61.6 & 30.8 & 151.3 & 0.27 \\
\hline 18 & Maternity/repro & 54.7 & 53.8 & 25.1 & 151.3 & 0.31 \\
\hline & Neonate conditions & 13.9 & 13.8 & 0.3 & 53.2 & 0.53 \\
\hline 20 & Poisoning & 12.3 & 12.5 & 4.2 & 24.5 & 0.28 \\
\hline & Healthy individuals & 21.7 & 21.5 & 4.2 & 90.1 & 0.51 \\
\hline & Social care needs & 25.1 & 24.5 & -80.4 & 140.1 & 0.85 \\
\hline & Other areas & 154.7 & 156.8 & 98.2 & 574.2 & 0.29 \\
\hline & GMS / PMS * & 126.9 & 128.8 & 90.8 & 237.4 & 0.14 \\
\hline & Total expenditure & 1183.1 & 1188.1 & 820.2 & 1705.9 & 0.13 \\
\hline \multicolumn{7}{|c|}{$\begin{array}{l}\text { NB Descriptive statistics across PCTs are unweighted and, for any given PCT, its } \\
\text { expenditure per head figures reflect its raw population adjusted for unavoidable cost } \\
\text { variations. The coefficient of variation (CV) is a measure of dispersion and is } \\
\text { calculated as the standard deviation divided by the mean. *The GMS/PMS figures exclude } \\
\text { three PCTs for whom the reported expenditure figures are either zero or implausibly } \\
\text { low. }\end{array}$} \\
\hline
\end{tabular}


Table 2. Results for cancer programme of care

\begin{tabular}{|c|c|c|c|c|c|c|c|c|}
\hline \multirow[t]{2}{*}{$\mathrm{N}=295$} & \multicolumn{2}{|c|}{$\begin{array}{l}\text { OLS } \\
(1)\end{array}$} & \multicolumn{2}{|c|}{$\begin{array}{l}\text { 2SLS } \\
(2)\end{array}$} & \multicolumn{2}{|c|}{$\begin{array}{l}\text { 2SLS } \\
\text { (3) }\end{array}$} & \multicolumn{2}{|c|}{$\begin{array}{l}\text { OLS } \\
(4)\end{array}$} \\
\hline & $\begin{array}{l}\text { Cancer } \\
\text { deaths }\end{array}$ & $\begin{array}{c}\text { Cancer } \\
\text { expenditure }\end{array}$ & $\begin{array}{l}\text { Cancer } \\
\text { deaths }\end{array}$ & $\begin{array}{c}\text { Cancer } \\
\text { expenditure }\end{array}$ & $\begin{array}{l}\text { Cancer } \\
\text { SYLL }\end{array}$ & $\begin{array}{c}\text { Cancer } \\
\text { expenditure }\end{array}$ & $\begin{array}{l}\text { Cancer } \\
\text { SYLL }\end{array}$ & $\begin{array}{c}\text { Cancer } \\
\text { expenditure }\end{array}$ \\
\hline Constant & $4.966(.103)$ & $-.546(1.171)$ & $6.919(.419)$ & $.751(1.267)$ & $6.712(.364)$ & $.725(1.302)$ & $5.234(.102)$ & $-1.027(1.175)$ \\
\hline Need & $.684(.034)$ & $.305(.167)$ & $.916(.068)$ & $.588(.197)$ & $.845(.059)$ & $.654(.212)$ & $0.670(.033)$ & $.239(.171))$ \\
\hline Cancer expenditure & $-.038(.024)$ & & $-.491(.097)$ & & $-.378(.085)$ & & $-0.035(.023)$ & \\
\hline Total Budget & & $.933(.153)$ & & $.874(.155)$ & & $.877(.159)$ & & $.956(.154)$ \\
\hline Non-cancer deaths & & $-.383(.071)$ & & $-.576(.099)$ & & & & \\
\hline Non-cancer SYLL & & & & & & $-.556(.100)$ & & $-.303(.066)$ \\
\hline Test statistics: & & & & & & & & \\
\hline Sargan $\left(\chi_{1}^{2}\right)$ & & & 1.575 (.210) & $.314(.575)$ & 2.750 (.097) & $1.357(.244)$ & & \\
\hline Anderson $\left(\chi_{2}^{2}\right)$ & & & $42 . .23(.000)$ & $214.2(.000)$ & $42.23(.000)$ & $177.4(.000)$ & & \\
\hline Cragg-Donald & & & $22.39(<.05)$ & $154.7(<.05)$ & $22.39(<.05)$ & $119.6(<.05)$ & & \\
\hline Partial $\mathrm{R}^{2}$ & & & .133 & .516 & .133 & .452 & & \\
\hline $\begin{array}{l}\text { Reset: } \\
\mathrm{F}(3,289)\end{array}$ & $11.29(.000)$ & & & & & & $6.16(.000)$ & \\
\hline $\mathrm{F}(3,288)$ & & $1.68(.171)$ & & & & & & $1.57(.195)$ \\
\hline Pesaran-Taylor $\left(\chi_{3}^{2}\right)$ & & & .15 (.985) & .33 (.954) & .03 (.998) & .03 (.999) & & \\
\hline Endogeneity $\left(\chi_{1}^{2}\right)$ : & & & $55.88(.000)$ & & $32.63(.000)$ & & & \\
\hline Cancer expenditure & & & & $7.92(.005)$ & & & & \\
\hline $\begin{array}{l}\text { Non-cancer deaths } \\
\text { Non-cancer SYLL }\end{array}$ & & & & & & $12.18(.000)$ & & \\
\hline
\end{tabular}

Note: Parentheses show standard errors for parameter estimates and p-values for the statistics. The instrument set for cancer expenditure consists of the proportion of households that are lone pensioner household and the proportion of the population providing unpaid care. 
Table 3. Results for circulatory diseases programme of care

\begin{tabular}{|c|c|c|c|c|c|c|c|c|}
\hline \multirow[t]{2}{*}{$\mathrm{N}=295$} & \multicolumn{2}{|c|}{$\begin{array}{l}\text { OLS } \\
(1)\end{array}$} & \multicolumn{2}{|c|}{$\begin{array}{l}\text { 2SLS } \\
(2)\end{array}$} & \multicolumn{2}{|c|}{$\begin{array}{l}\text { 2SLS } \\
\text { (3) }\end{array}$} & \multicolumn{2}{|c|}{$\begin{array}{l}\text { OLS } \\
(4)\end{array}$} \\
\hline & $\begin{array}{c}\text { Circulatory } \\
\text { deaths }\end{array}$ & $\begin{array}{l}\text { Circulatory } \\
\text { expenditure }\end{array}$ & $\begin{array}{c}\text { Circulatory } \\
\text { deaths }\end{array}$ & $\begin{array}{l}\text { Circulatory } \\
\text { expenditure }\end{array}$ & $\begin{array}{l}\text { Circulatory } \\
\text { SYLL }\end{array}$ & $\begin{array}{l}\text { Circulatory } \\
\text { expenditure }\end{array}$ & $\begin{array}{c}\text { Circulatory } \\
\text { SYLL }\end{array}$ & $\begin{array}{l}\text { Circulatory } \\
\text { expenditure }\end{array}$ \\
\hline Constant & $6.492(.245)$ & $1.072(.911)$ & $11.23(.728)$ & $4.49(1.242)$ & $11.57(.766)$ & $6.63(1.633)$ & $6.751(.267)$ & $1.026(.942)$ \\
\hline Need & $1.595(.068)$ & $.606(.115)$ & $2.450(.153)$ & $1.069(.161)$ & $2.652(.161)$ & $1.283(.203)$ & $1.781(.074)$ & $0.579(.116)$ \\
\hline Circulatory expenditure & $-.402(.051)$ & & $-1.387(.151)$ & & $-1.427(.159)$ & & $-0.424(.055)$ & \\
\hline Total Budget & & $.804(.109)$ & & $.764(.118)$ & & $.716(.130)$ & & $0.798(.110)$ \\
\hline Non-circulatory deaths & & $-.400(.092)$ & & $-1.052(.176)$ & & & & \\
\hline Non-circulatory SYLL & & & & & & $-1.349(.239)$ & & $-0.361(.093)$ \\
\hline \% White ethnic group & & $.372(.050)$ & & $.369(.054)$ & & $.365(.059)$ & & $0.371(.050)$ \\
\hline \multicolumn{9}{|l|}{ Test statistics: } \\
\hline Sargan $\left(\chi_{2}^{2}\right)$ & & & $4.113(.128)$ & $4.273(.118)$ & $5.034(.081)$ & $1.699(.428)$ & & \\
\hline Anderson $\left(\chi_{3}^{2}\right)$ & & & $86.92(.000)$ & $111.4(.000)$ & $86.92(.000)$ & $68.93(.000)$ & & \\
\hline Cragg-Donald & & & $33.12(<.05)$ & $44.04(<.05)$ & $33.12(<.05)$ & $25.27(<.05)$ & & \\
\hline Partial $\mathrm{R}^{2}$ & & & .255 & .315 & .255 & .208 & & \\
\hline $\begin{array}{l}\text { Reset: } \\
F(3,289)\end{array}$ & $2.09(.035)$ & & & & & & $2.45(.063)$ & \\
\hline $\mathrm{F}(3,287)$ & & $2.17(.092)$ & & & & & & $2.81(.040)$ \\
\hline Pesaran-Taylor $\left(\chi_{3}^{2}\right)$ & & & $6.78(.08)$ & $0.42(.936)$ & $4.45(.217)$ & $1.33(.723)$ & & \\
\hline \multicolumn{9}{|l|}{ Endogeneity $\left(\chi_{1}^{2}\right)$ : } \\
\hline Circulatory expenditure & & & $129.5(.000)$ & & $112.4(.000)$ & & & \\
\hline Non-circulatory deaths & & & & $23.48(.000)$ & & & & \\
\hline Non-circulatory YLL & & & & & & $29.75(.000)$ & & \\
\hline
\end{tabular}

\footnotetext{
Note: Parentheses show standard errors for parameter estimates and p-values for the statistics. Instrument set for cancer expenditure consists of the proportion of households that are one pensioner household, the proportion of the population providing unpaid care and the population weighted index of multiple deprivation based on ward level IMD 2000 scores. The number of observations is 295 not 303 . There are 8 missing PCTs because the variables used in the regression models were constructed at slightly different dates and between these dates there were a small number of PCT boundary changes.
} 
Figure 1 PCT spend (cost adjusted) per person on cancer $£$

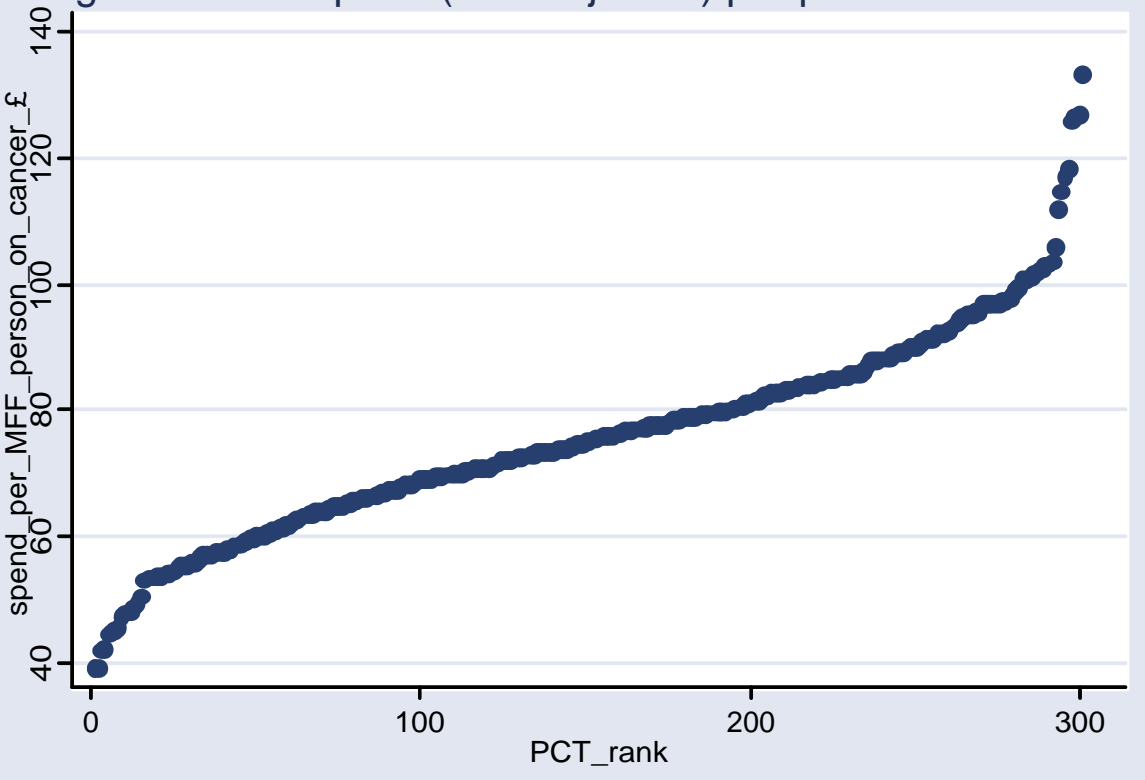

Figure 2 PCT spend (cost adjusted) per person on CHD $£$

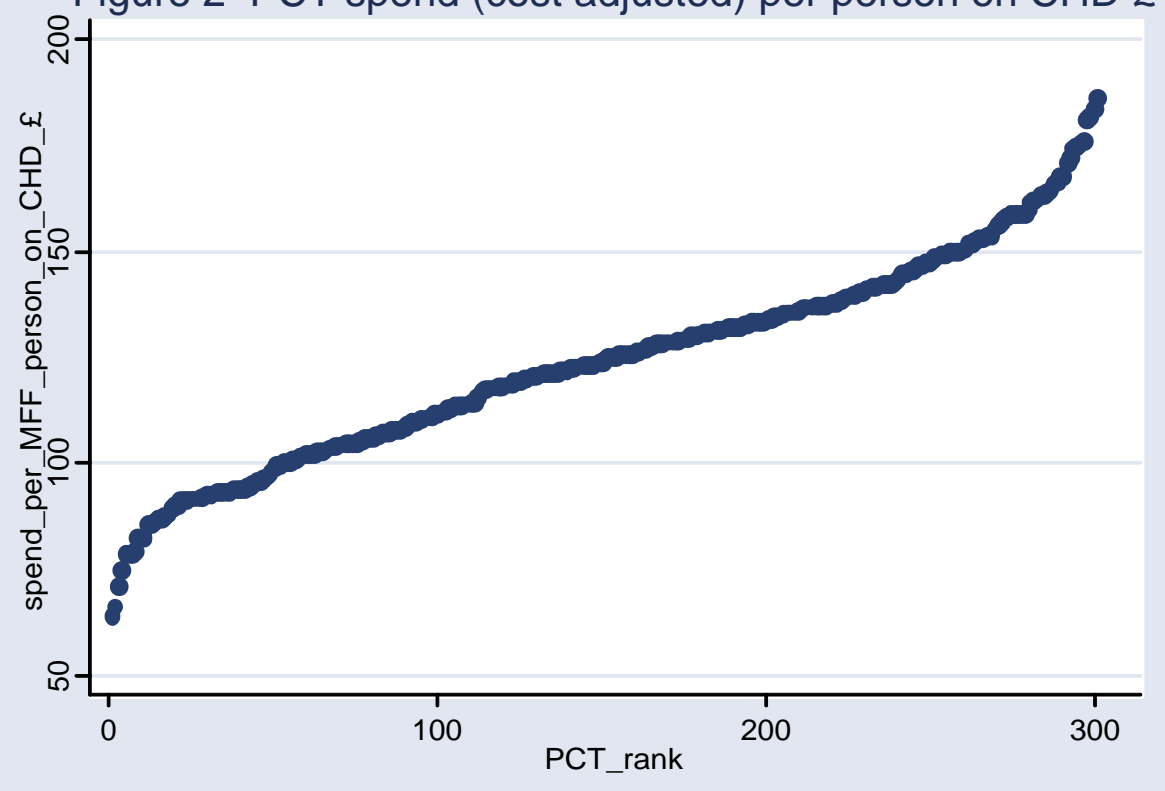




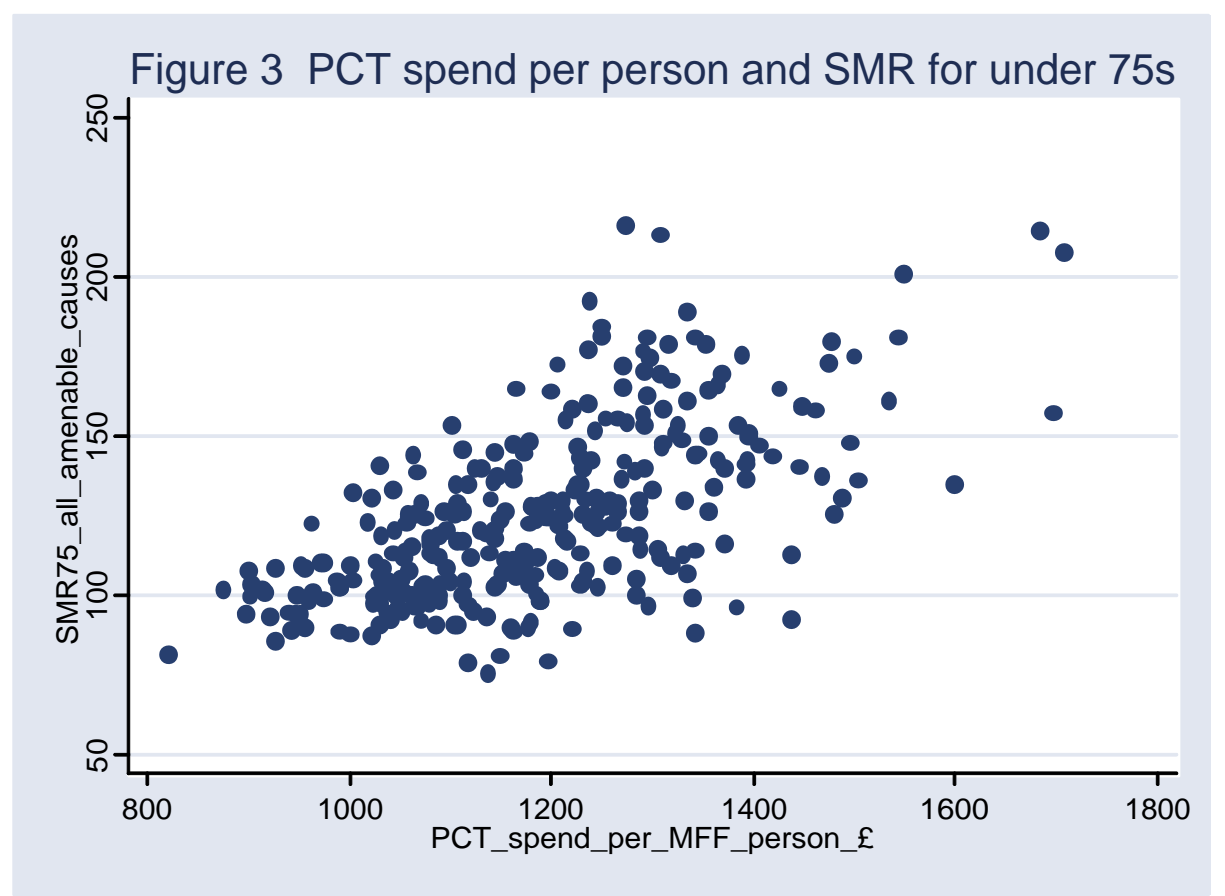

Figure 4 PCT spend per person and PCT SMR for under 75s

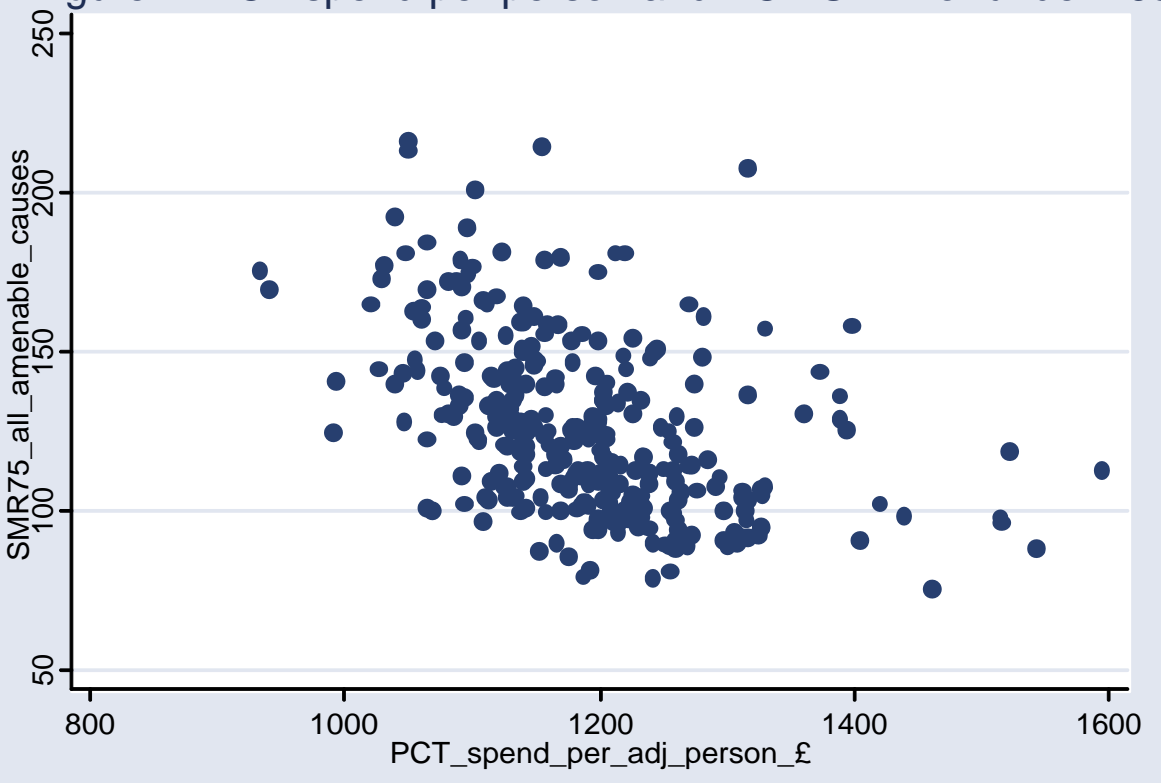




\section{APPENDIX}

\section{Table A1 Deaths considered amenable to health care}

Deaths considered amenable to health care are defined as those from the following causes for the specific age groups stated. See http://www.nchod.nhs.uk/ for further details.

Intestinal infections (ICD-10 A00-A09, ICD-9 001-009), ages 0-14 years;

Tuberculosis (ICD-10 A15-A19, B90; ICD-9 010-018, 137), ages 0-74 years;

Other infectious diseases (diptheria, tetanus, poliomyelitis) (ICD-10 A36, A35, A80; ICD-9 032, 037, 045), ages

$0-74$ years;

Whooping cough (ICD-10 A37, ICD-9 033), ages 0-14 years;

Septicaemia (ICD-10 A40-A41, ICD-9 038), ages 0-74 years;

Measles (ICD-10 B05, ICD-9 055), ages 1-14 years;

Malignant neoplasm of colon and rectum (ICD-10 C18-C21, ICD-9 153-154), ages 0-74 years;

Malignant neoplasm of skin (ICD-10 C44, ICD-9 173), ages 0-74 years;

Malignant neoplasm of female breast (ICD-10 C50, ICD-9 174), ages 0-74 years;

Malignant neoplasm of cervix uteri (ICD-10 C53, ICD-9 180), ages 0-74 years;

Malignant neoplasm of unspecified part of the uterus (ICD-10 C54-C55, ICD-9 179, 182), ages 0-44 years;

Malignant neoplasm of testis (ICD-10 C62, ICD-9 186), 0-74 years;

Hodgkin's disease (ICD-10 C81, ICD-9 201), ages 0-74 years;

Leukaemia (ICD-10 C91-C95, ICD-9 204-208), ages 0-44 years;

Diseases of the thyroid (ICD-10 E00-E07, ICD-9 240-246), ages 0-74 years;

Diabetes mellitus (ICD-10 E10-E14, ICD-9 250), ages 0-49 years;

Epilepsy (ICD-10 G40-G41, ICD-9 345), 0-74 years;

Chronic rheumatic heart disease (ICD-10 I05-I09, ICD-9 393-398), ages 0-74 years;

Hypertensive disease (ICD-10 I10-I13, I15 ; ICD-9 401-405), ages 0-74 years;

Ischaemic heart disease (ICD-10 I20-I25, ICD-9 410-414), ages 0-74 years;

Cerebrovascular disease (ICD-10 I60-I69, ICD-9 430-438), ages 0-74 years;

All respiratory diseases (excl. pneumonia, influenza and asthma) (ICD-10 J00-J09, J20-J44, J47-J99; ICD-9 460479, 488-492, 494-519), ages 1-14 years;

Influenza (ICD-10 J10-J11, ICD-9 487), ages 0-74 years;

Pneumonia (ICD-10 J12-J18, ICD-9 480-486), ages 0-74 years;

Asthma (ICD-10 J45-J46, ICD-9 493), ages 0-44 years;

Peptic ulcer (ICD-10 K25-K27, ICD-9 531-533), ages 0-74 years;

Appendicitis (ICD-10 K35-K38, ICD-9 540-543), ages 0-74 years;

Abdominal hernia (ICD-10 K40-K46, ICD-9 550-553), ages 0-74 years;

Cholelithiasis \& cholecystitis (ICD-10 K80-K81, ICD-9 574-575.1), ages 0-74 years;

Nephritis and nephrosis (ICD-10 N00-N07, N17-N19, N25-N27; ICD-9 580-589), ages 0-74 years;

Benign prostatic hyperplasia (ICD-10 N40, ICD-9 600), ages 0-74 years;

Maternal deaths (ICD-10 O00-O99, ICD-9 630-676), ages 0-74 years;

Congenital cardiovascular anomalies (ICD-10 Q20-Q28, ICD-9 745-747), ages 0-74 years;

Perinatal deaths (all causes excl. stillbirths), ages 0-6 days;

Misadventures to patients during surgical and medical care (ICD-10 Y60-Y69, Y83-Y84; ICD-9 E870-E876,

E878-E879), ages 0-74 years. 
Table A2

First-stage regressions for cancer 2SLS results presented in Table 2

\begin{tabular}{|c|c|c|c|}
\hline & $\begin{array}{l}\text { Cancer } \\
\text { expenditure }\end{array}$ & $\begin{array}{l}\text { Non-cancer } \\
\text { deaths (SMR) }\end{array}$ & $\begin{array}{l}\text { Non-cancer } \\
\text { deaths (SYLL) }\end{array}$ \\
\hline Constant & $5.265(.255)$ & 3.497 (.651) & $3.881(.754)$ \\
\hline Need & $0.295(.084)$ & $1.732(.081)$ & $1.963(.094)$ \\
\hline$\%$ lone pensioner & $0.626(.099)$ & $-0.738(.056)$ & $-0.671(.065)$ \\
\hline \% unpaid carers & $-0.111(.121)$ & $-0.255(.069)$ & $-0.391(.079)$ \\
\hline Total budget & & $-0.135(.091)$ & $-0.194(.106)$ \\
\hline \multicolumn{4}{|l|}{ Test statistic } \\
\hline \multicolumn{4}{|c|}{ Joint test coefficients: } \\
\hline$F(2,291)$ & $22.39(\mathrm{p}<0.05)$ & & \\
\hline $\mathrm{F}(2,290)$ & & $154.67(\mathrm{p}<0.05)$ & $119.5(\mathrm{p}<0.05)$ \\
\hline
\end{tabular}

Notes: the test statistic is an F statistic testing the hull hypothesis that the coefficients on the instruments that are excluded from the second-stage regression are equal to zero. $\%$ lone pensioner $=$ the proportion of households that are lone pensioner households and $\%$ unpaid carers $=$ the proportion of the population providing unpaid care (according to the 2001 Census, a person is a provider of unpaid care if they give any help or support to family members, friends, neighbours or others because of longterm physical or mental ill-health or disability, or problems relating to old age). 
Table A3

First-stage regressions for circulatory 2SLS results presented in Table 3

\begin{tabular}{|c|c|c|c|}
\hline & $\begin{array}{l}\text { Circulatory } \\
\text { expenditure }\end{array}$ & $\begin{array}{l}\text { Non-circulatory } \\
\text { deaths (SMR) }\end{array}$ & $\begin{array}{l}\text { Non-circulatory } \\
\text { deaths (SYLL) }\end{array}$ \\
\hline Constant & $6.391(.183)$ & $3.445(.444)$ & $4.312(.471)$ \\
\hline Need & $0.767(.231)$ & $0.662(.125)$ & $0.669(.133)$ \\
\hline \% lone pensioner & $0.452(.084)$ & $-0.337(.046)$ & $-0.238(.049)$ \\
\hline \% unpaid carers & $0.240(.094)$ & $-0.140(.052)$ & $-0.158(.056)$ \\
\hline IMD2000 & $-0.049(.057)$ & $0.060(.029)$ & $0.050(.031)$ \\
\hline Total budget & & $0.029(.061)$ & $-0.030(.065)$ \\
\hline$\%$ white ethnic group & & $0.238(.034)$ & $0.196(.037)$ \\
\hline \multicolumn{4}{|l|}{ Test statistic } \\
\hline \multicolumn{4}{|l|}{ Joint test coefficients: } \\
\hline$F(3,290)$ & $33.12(\mathrm{p}<0.05)$ & & \\
\hline $\mathrm{F}(3,288)$ & & $44.04 \quad(\mathrm{p}<0.05)$ & $25.27(\mathrm{p}<0.05)$ \\
\hline
\end{tabular}

Notes: the test statistic is an F statistic testing the hull hypothesis that the coefficients on the instruments that are excluded from the second-stage regression are equal to zero. \% white ethnic group $=$ proportion of the population in a 'white' ethnic group, $\%$ lone pensioner $=$ the proportion of households that are lone pensioner households, and \% unpaid carers = the proportion of the population providing unpaid care (according to the 2001 Census, a person is a provider of unpaid care if they give any help or support to family members, friends, neighbours or others because of long-term physical or mental ill-health or disability, or problems relating to old age). 